Mathematical Research Letters 9, 95-103 (2002)

\title{
MINIMAL HYPERSURFACES WITH FINITE INDEX
}

\author{
Peter Li ${ }^{1}$ AND JiAPing WANG ${ }^{2}$
}

\section{$\S 0$ Introduction}

In an article of Cao-Shen-Zhu [C-S-Z], they proved that a complete, immersed, stable minimal hypersurface $M^{n}$ of $\mathbb{R}^{n+1}$ with $n \geq 3$ must have only one end. When $n=2$, it was proved independently by do Carmo-Peng [dC-P] and FischerColbrie-Schoen [FC-S] that a complete, immersed, oriented stable minimal surface in $\mathbb{R}^{3}$ must be a plane. Later Gulliver $[\mathrm{G}]$ and Fischer-Colbrie $[\mathrm{FC}]$ proved that if a complete, immersed, minimal surface in $\mathbb{R}^{3}$ has finite index, then it must be conformally equivalent to a compact Riemann surface with finitely many punctures. Fischer-Colbrie actually proved this for minimal surfaces in a complete three dimensional manifold with non-negative scalar curvature. In any event, a corollary is that if a complete, immersed, oriented minimal surface in $\mathbb{R}^{3}$ has finite index then it must have finitely many ends. The purpose of this paper is to generalize this result for finitely many ends to higher dimensional minimal hypersurfaces in Euclidean space (see Theorem 5). In fact, we will also show that the first $L^{2}$-Betti number of such a manifold must be finite.

The strategy of Cao-Shen-Zhu was to utilize a result of Schoen-Yau [S-Y] asserting that a complete, stable minimal hypersurface of $\mathbb{R}^{n+1}$ cannot admit a non-constant harmonic function with finite Dirichlet integral. Assuming that $M$ has more than one end, Cao-Shen-Zhu constructed a non-constant harmonic function with finite Dirichlet integral. This approach very much fits into the scheme studied by the first author and Tam in [L-T2]. In fact, the authors showed that the number of non-parabolic ends of any complete Riemannian manifold is bounded above by the dimension of the space of bounded harmonic functions with finite Dirichlet integral. The proof of Cao-Shen-Zhu can be modified to show that each end of a complete, immersed, minimal submanifold must be nonparabolic. Due to this connection with harmonic functions, our approach is to refine the argument of Schoen-Yau to obtain an estimate of the dimension of the space of harmonic functions with finite Dirichlet integral. Unfortunately, our estimate depends on the geometry of $M$ on a compact subset, whose existence is guaranteed by the finite index assumption. While we succeeded in proving

\footnotetext{
Received September 26, 2001.

${ }^{1}$ Research partially supported by NSF grant \#DMS-9971418 and an Earmarked grant of Hong Kong.

${ }^{2}$ Research partially supported by NSF grant \#DMS-0072181.
} 
finite index implies finitely many ends, it is unclear if one can actually estimate the number of ends by the index directly. This will be an interesting issue to investigate in the future.

\section{$\S 1$ Preliminaries}

Let us first recall (see [L-T2] and [L3]) that an end $E$ of a complete manifold $M$ is non-parabolic means that $E$ admits a positive Green's function with Neumann boundary condition. First, we will recall a theorem of the first author and Tam in $[\mathrm{L}-\mathrm{T} 2]$.

Theorem 1 (Li-Tam). Let $M$ be a complete Riemannian manifold. Let $\mathcal{H}_{D}^{0}(M)$ denote the space of bounded harmonic functions with finite Dirichlet integral. Then the number of non-parabolic ends of $M$ is at most the dimension of $\mathcal{H}_{D}^{0}(M)$.

Observe that if $u$ is a harmonic function with finite Dirichlet integral then its exterior differential $d u$ is an $L^{2}$ harmonic 1-form. Moreover, $d u=0$ if and only if $u$ is identically constant. Hence

$$
\operatorname{dim} \mathcal{H}_{D}^{0}(M) \leq \operatorname{dim} H^{1}\left(L^{2}(M)\right)+1
$$

Using this inequality, we can state Theorem 1 in terms of the first $L^{2}$ Betti number.

Corollary 2. Let $M$ be a complete Riemannian manifold. Let $H^{1}\left(L^{2}(M)\right)$ be the first $L^{2}$-cohomology of $M$. Then the number of non-parabolic ends of $M$ is bounded from above by $\operatorname{dim} H^{1}\left(L^{2}(M)\right)+1$.

This corollary enables us to estimate the number of ends of a minimal hypersurface if we can show that all its ends are non-parabolic. In fact, it was proved in [C-S-Z] that this is the case for minimal submanifolds of dimension at least three in $\mathbb{R}^{N}$. For completeness sake, we provide a presentation which extracts the main points of the proof and state it for more general situations (Corollary 4) in terms of non-parabolicity.

Theorem 3 (Cao-Shen-Zhu). Let $M^{n}$ be a complete, immerse, minimal submanifold of $\mathbb{R}^{N}$. If $n \geq 3$, then each end of $M$ must be non-parabolic.

Proof. Let $E$ be an end of $M$. For $R$ sufficiently large, let us consider the set $E_{R}=E \cap B_{p}(R)$, where $B_{p}(R)$ is the geodesic ball of radius $R$ in $M$ centered at some point $p \in M$. Let us denote by $r$ the distance function of $M$ to the point $p$. Suppose the function $f_{R}$ is the solution of the equation

$$
\begin{array}{cc}
\Delta f_{R}=0 & \text { on } E_{R}, \\
f_{R}=1 & \text { on } \partial E
\end{array}
$$


and

$$
f_{R}=0 \quad \text { on } E \cap \partial B_{p}(R) .
$$

By the maximum principle, $f_{R}$ is uniformly bounded between 0 and 1 . This bound and the gradient estimate imply that the sequence $f_{R}$ converges uniformly on compact subsets of $E$ to a harmonic function $f$ with boundary condition

$$
f=1 \quad \text { on } \partial E \text {. }
$$

Moreover, $f$ will satisfy the bounds

$$
0 \leq f \leq 1
$$

If we can show that $f$ is non-constant, then $E$ will be non-parabolic (see [L-T1] and [L3]).

For a fixed $0<R_{0}<R$ such that $E_{R_{0}} \neq \emptyset$, let $\phi$ be a non-negative cut-off function satisfying the properties that

$$
\begin{gathered}
\phi=1 \quad \text { on } E_{R} \backslash E_{R_{0}}, \\
\phi=0 \quad \text { on } \partial E,
\end{gathered}
$$

and

$$
|\nabla \phi| \leq C_{1}
$$

The Sobolev inequality of Michael-Simon [M-S], integration by parts, and the fact that $f_{R}$ is harmonic, imply that

$$
\begin{aligned}
\left(\int_{E_{R}}\left(\phi f_{R}\right)^{\left.\frac{2 n}{n-2}\right)^{\frac{n-2}{n} \leq} \leq}\right. & C \int_{E_{R}}\left|\nabla\left(\phi f_{R}\right)\right|^{2} \\
= & C\left(\int_{E_{R}}|\nabla \phi|^{2} f_{R}^{2}+2 \int_{E_{R}} \phi f_{R}\left\langle\nabla \phi, \nabla f_{R}\right\rangle\right. \\
& \left.+\int_{E_{R}} \phi^{2}\left|\nabla f_{R}\right|^{2}\right) \\
= & C\left(\int_{E_{R}}|\nabla \phi|^{2} f_{R}^{2}+\frac{1}{2} \int_{E_{R}}\left\langle\nabla\left(\phi^{2}\right), \nabla\left(f_{R}^{2}\right)\right\rangle\right. \\
& \left.+\int_{E_{R}} \phi^{2}\left|\nabla f_{R}\right|^{2}\right) \\
= & C \int_{E_{R}}|\nabla \phi|^{2} f_{R}^{2} .
\end{aligned}
$$

In particular, for a fixed $R_{1}$ satisfying $R_{0}<R_{1}<R$, we have

$$
\left(\int_{E_{R_{1}} \backslash E_{R_{0}}} f_{R}^{\frac{2 n}{n-2}}\right)^{\frac{n-2}{n}} \leq C_{2} \int_{E_{R_{0}}} f_{R}^{2} .
$$


If the limiting function $f$ is identically constant, then $f$ must be identically 1 because of its boundary condition. Letting $R \rightarrow \infty$, we obtain

$$
\left(V_{E}\left(R_{1}\right)-V_{E}\left(R_{0}\right)\right)^{\frac{n-2}{n}} \leq C V_{E}\left(R_{0}\right)
$$

where $V_{E}(r)$ denotes the volume of the set $E_{r}$. Since $R_{1}>R_{0}$ is arbitrary, this implies that $E$ must have finite volume. However, since an end of a minimal submanifold must have infinite volume, this contradicts the assumption that $f=1$, and the theorem is proved.

It is clear in the above argument that this theorem can be generalized to an arbitrary Riemannian manifold.

Corollary 4. Let $E$ be an end of a complete Riemannian manifold. Suppose for some $\nu \geq 1, E$ satisfies a Sobolev type inequality of the form

$$
\left(\int_{E}|u|^{2 \nu}\right)^{\frac{1}{\nu}} \leq C \int_{E}|\nabla u|^{2}
$$

for all compactly supported function $u \in W_{1,2}(E)$ defined on $E$, then $E$ must either have finite volume or be non-parabolic.

We would like to remark that it was proved independently by Grigor'yan [Gr] and Varopoulos [V] that if a manifold is non-parabolic then its volume growth must satisfy

$$
\int_{1}^{\infty} \frac{t d t}{V_{p}(t)}<\infty
$$

In particular, when combined with Corollary 4, this implies that if an end satisfies a Sobolev type inequality as hypothesized in Corollary 4, then it must either have finite volume or its volume growth must be at least quadratic satisfying (1.1).

\section{$\S 2$ Proof of Main Theorem}

We are now ready to prove our main result.

Theorem 5. Let $M^{n}$ be a complete, immersed, oriented minimal hypersurface in $\mathbb{R}^{n+1}$ with $n \geq 3$. Suppose $M$ has finite index. Then $M$ must have finite first $L^{2}$-Betti number, i.e. $\operatorname{dim} H^{1}\left(L^{2}(M)\right)<\infty$. In particular, $M$ must have finitely many ends.

Proof. The assumption that $M$ has finite index implies that there exists a compact set $\Omega \subset M$ such that $M \backslash \Omega$ is stable. In particular, we may assume that $\Omega \subset B_{p}\left(R_{0}\right)$ for some geodesic ball centered at $p \in M$ of radius $R_{0}$. The monotonicity of eigenvalues implies that $M \backslash B_{p}\left(R_{0}\right)$ is stable. In particular, if $|A|^{2}$ 
denotes the square of the length of the second fundamental form of $M$, then the stability inequality $[\mathrm{S}-\mathrm{Y}]$ asserts that

$$
\int_{M \backslash B_{p}\left(R_{0}\right)} \psi^{2}|A|^{2} \leq \int_{M \backslash B_{p}\left(R_{0}\right)}|\nabla \psi|^{2}
$$

for all compactly supported function $\psi$ on $M \backslash B_{p}\left(R_{0}\right)$.

For any $L^{2}$ harmonic 1-form $\omega$ defined on $M$, let us denote

$$
h=|\omega|
$$

to be the length of the $\omega$. The Bochner formula (see [L2]) asserts that

$$
\Delta h^{2} \geq 2 \operatorname{Ric}(\omega, \omega)+2|\nabla \omega|^{2},
$$

where Ric denotes the Ricci curvature of $M$ and $\nabla \omega$ is the covariant derivative of $\omega$. Using the Gauss curvature equation, we conclude that

$$
\operatorname{Ric}(\omega, \omega) \geq-|A|^{2} h^{2} .
$$

Since $\omega$ is an $L^{2}$ harmonic 1 -form, it must be both closed and co-closed. In particular, in terms of an orthonormal co-frame $\left\{\omega_{1}, \ldots, \omega_{n}\right\}$, we can write $\omega=$ $a_{i} \omega_{i}$. Then the closed condition is given by

$$
a_{i, j}=a_{j, i}
$$

and the co-closed condition is given by

$$
\sum_{i=1}^{n} a_{i, i}=0 .
$$

On the other hand,

$$
\begin{aligned}
|\nabla \omega|^{2} & =\sum_{i, j} a_{i, j}^{2} \\
& \geq \sum_{j=1}^{n} a_{1, j}^{2}+\sum_{\alpha=2}^{n} a_{\alpha, 1}^{2}+\sum_{\alpha=2}^{n} a_{\alpha, \alpha}^{2} \\
& \geq \sum_{j=1}^{n} a_{1, j}^{2}+\sum_{\alpha=2}^{n} a_{\alpha, 1}^{2}+\frac{1}{n-1}\left(\sum_{\alpha=2}^{n} a_{\alpha, \alpha}\right)^{2} .
\end{aligned}
$$

Using both the closed and co-closed conditions, we conclude that

$$
|\nabla \omega|^{2} \geq \frac{n}{n-1} \sum_{j=1}^{n} a_{1, j}^{2} .
$$


However, at any fixed point $x \in M$, if we choose an orthonormal co-frame such that $|\omega| \omega_{1}=\omega$, then

$$
\begin{aligned}
\left|\nabla\left(h^{2}\right)\right|^{2} & =4 \sum_{j=1}^{n}\left(a_{1} a_{1, j}\right)^{2} \\
& \leq 4 h^{2} \sum_{j=1}^{n} a_{1, j}^{2} .
\end{aligned}
$$

Combining with (2.2), (2.3), and (2.4), we obtain

$$
\Delta h \geq-|A|^{2} h+\frac{|\nabla h|^{2}}{(n-1) h} .
$$

By choosing $\psi=\phi h$ with $\phi$ being a non-negative compactly supported function on $M \backslash B_{p}\left(R_{0}\right),(2.1)$ becomes

$$
\begin{aligned}
\int_{M \backslash B_{p}\left(R_{0}\right)} \phi^{2}|A|^{2} h^{2} \leq & \int_{M \backslash B_{p}\left(R_{0}\right)}|\nabla \phi|^{2} h^{2}+2 \int_{M \backslash B_{p}\left(R_{0}\right)} \phi h\langle\nabla \phi, \nabla h\rangle \\
& +\int_{M \backslash B_{p}\left(R_{0}\right)} \phi^{2}|\nabla h|^{2} \\
= & \int_{M \backslash B_{p}\left(R_{0}\right)}|\nabla \phi|^{2} h^{2}-\int_{M \backslash B_{p}\left(R_{0}\right)} \phi^{2} h \Delta h .
\end{aligned}
$$

Combining with (2.5), we have

$$
\int_{M \backslash B_{p}\left(R_{0}\right)} \phi^{2}|\nabla h|^{2} \leq(n-1) \int_{M \backslash B_{p}\left(R_{0}\right)}|\nabla \phi|^{2} h^{2} .
$$

On the other hand, the Sobolev inequality for minimal submanifold [M-S] implies that

$$
\begin{aligned}
\left(\int_{M \backslash B_{p}\left(R_{0}\right)}(\phi h)^{\frac{2 n}{n-2}}\right)^{\frac{n-2}{n}} & \leq C \int_{M \backslash B_{p}\left(R_{0}\right)}|\nabla(\phi h)|^{2} \\
& \leq 2 C \int_{M \backslash B_{p}\left(R_{0}\right)} \phi^{2}|\nabla h|^{2}+2 C \int_{M \backslash B_{p}\left(R_{0}\right)}|\nabla \phi|^{2} h^{2} .
\end{aligned}
$$

Combining with (2.6), we obtain

$$
\left(\int_{M \backslash B_{p}\left(R_{0}\right)}(\phi h)^{\frac{2 n}{n-2}}\right)^{\frac{n-2}{n}} \leq 2 n C \int_{M \backslash B_{p}\left(R_{0}\right)}|\nabla \phi|^{2} h^{2} .
$$


For $R>R_{0}+1$, let us choose $\phi$ satisfying the properties that

$$
\begin{gathered}
\phi= \begin{cases}0 & \text { on } B_{p}\left(R_{0}\right) \\
1 & \text { on } B_{p}(R) \backslash B_{p}\left(R_{0}+1\right) \\
0 & \text { on } M \backslash B_{p}(2 R),\end{cases} \\
|\nabla \phi| \leq C_{3} \quad \text { on } B_{p}\left(R_{0}+1\right) \backslash B_{p}\left(R_{0}\right)
\end{gathered}
$$

and

$$
|\nabla \phi| \leq C_{3} R^{-1} \quad \text { on } B_{p}(2 R) \backslash B_{p}(R)
$$

for some constant $C_{3}>0$. Applying this to (2.7), we have

$$
\begin{aligned}
\left(\int_{B_{p}(R) \backslash B_{p}\left(R_{0}+1\right)} h^{\frac{2 n}{n-2}}\right)^{\frac{n-2}{n}} & \leq C_{4} \int_{B_{p}\left(R_{0}+1\right) \backslash B_{p}\left(R_{0}\right)} h^{2} \\
& +C_{4} R^{-2} \int_{B_{p}(2 R) \backslash B_{p}(R)} h^{2} .
\end{aligned}
$$

Using the assumption that $h$ is in $L^{2}$ and letting $R \rightarrow \infty$, the second term tends to 0 and we conclude that

$$
\left(\int_{M \backslash B_{p}\left(R_{0}+1\right)} h^{\frac{2 n}{n-2}}\right)^{\frac{n-2}{n}} \leq C_{4} \int_{B_{p}\left(R_{0}+1\right) \backslash B_{p}\left(R_{0}\right)} h^{2} .
$$

On the other hand, the Schwarz inequality asserts that

$$
\int_{B_{p}\left(R_{0}+2\right) \backslash B_{p}\left(R_{0}+1\right)} h^{2} \leq V_{p}^{\frac{2}{n}}\left(R_{0}+2\right)\left(\int_{B_{p}\left(R_{0}+2\right) \backslash B_{p}\left(R_{0}+1\right)} h^{\frac{2 n}{n-2}}\right)^{\frac{n-2}{n}} .
$$

Together with (2.8), we conclude that there exists a constant $C_{5}>0$ depending on $V_{p}\left(R_{0}+2\right)$ such that

$$
\int_{B_{p}\left(R_{0}+2\right)} h^{2} \leq C_{5} \int_{B_{p}\left(R_{0}+1\right)} h^{2} .
$$

The fact that $h$ satisfies the differential inequality (2.5) implies that we can apply the Moser iteration argument (see [L2]) and conclude that

$$
h^{2}(x) \leq C_{6} \int_{B_{x}(1)} h^{2}
$$

where $C_{6}>0$ depends only on $n$ and the upper bound of $|A|^{2}$ on $B_{x}(1)$. In particular, if $x \in B_{p}\left(R_{0}+1\right)$ has the property that

$$
h^{2}(x)=\sup _{B_{p}\left(R_{0}+1\right)} h^{2},
$$


then

$$
\sup _{B_{p}\left(R_{0}+1\right)} h^{2} \leq C_{6} \int_{B_{p}\left(R_{0}+2\right)} h^{2} .
$$

Combining with (2.9), this implies that there exists constant $C_{7}>$ depending only on $n, V_{p}\left(R_{0}+2\right)$, and $\sup _{B_{p}\left(R_{0}+2\right)}|A|^{2}$, such that

$$
\sup _{B_{p}\left(R_{0}+1\right)} h^{2} \leq C_{7} \int_{B_{p}\left(R_{0}+1\right)} h^{2}
$$

We are now ready to show that $H^{1}\left(L^{2}(M)\right)$ is finite dimensional. It suffices to show that any finite dimensional subspace $K$ of $H^{1}\left(L^{2}(M)\right)$ must have its dimension bounded by a fixed constant. Let $k$ be the dimension of $K$. Let us consider the bilinear form defined on $K$ given by

$$
\int_{B_{p}\left(R_{0}+1\right)}\langle\omega, \theta\rangle
$$

Note that if

$$
\int_{B_{p}\left(R_{0}+1\right)}|\omega|^{2}=0
$$

for some $\omega \in K$, then by the unique continuation property $\omega$ must be identically 0 . This implies that the bilinear form is an inner product defined on $K$.

According to Lemma 11 of [L1], there exists an $\omega \in K$ such that

$$
k \int_{B_{p}\left(R_{0}+1\right)}|\omega|^{2} \leq V_{p}\left(R_{0}+1\right)(\min \{n, k\}) \sup _{B_{p}\left(R_{0}+1\right)}|\omega|^{2} .
$$

However, combining with (2.10) we conclude that

$$
k \leq C_{8}
$$

with $C_{8}>0$ depending only on $n, V_{p}\left(R_{0}+2\right)$, and $\sup _{B_{p}\left(R_{0}+2\right)}|A|^{2}$. The theorem follows by applying Corollary 2 and Theorem 3.

\section{References}

[C-S-Z] H. Cao, Y. Shen and S. Zhu, The structure of stable minimal hypersurfaces in $\mathbb{R}^{n+1}$, Math. Res. Lett. 4 (1997), no. 5, 637-644.

[dC-P] M. do Carmo and C. K. Peng, Stable complete minimal surfaces in $\mathbb{R}^{3}$ are planes, Bull. Amer. Math. Soc. (N.S.) 1 (1979), no. 6, 903-906.

[FC] D. Fischer-Colbrie, On complete minimal surfaces with finite Morse index in three manifolds, Invent. Math. 82 (1985), no. 1, 121-132.

[FC-S] D. Fischer-Colbrie and R. Schoen, The structure of complete stable minimal surfaces in 3-manifolds of non-negative scalar curvature, Comm. Pure Appl. Math. 33 (1980), no. 2, 199-211. 
[Gr] A. Grigor'yan, Existence of the Green function on a manifold, (Russian) Uspekhi Mat. Nauk 38 (1983), no. 1 (229), 161-162.

[G] R. Gulliver, Index and total curvature of complete minimal surfaces, Geometric measure theory and the calculus of variations (Arcata, Calif., 1984), 207-211, Proc. Sympos. Pure Math., 44, Amer. Math. Soc., Providence, RI, 1986.

[L1] P. Li, On the Sobolev constant and the p-spectrum of a compact Riemannian manifold, Ann. Sci. École Norm. Sup. (4) 13 (1980), no. 4, 451-468.

[L2] P. Li, Lecture Notes on Geometric Analysis, Lecture Notes Series, 6, Seoul National University, Research Institute of Mathematics, Global Analysis Research Center, Seoul, 1993.

[L3] P. Li, Curvature and function theory on Riemannian manifolds, Survey in Differential Geometry "In Honor of Atiyah, Bott, Hirzebruch, and Singer", vol. VII, International Press, Cambridge, 2000, 71-111.

[L-T1] P. Li and L. F. Tam, Symmetric Green's functions on complete manifolds, Amer. J. Math. 109 (1987), no. 6, 1129-1154.

[L-T2] P. Li and L.F. Tam, Harmonic functions and the structure of complete manifolds, J. Differential Geom. 35 (1992), no. 2, 359-383.

[M-S] J. H. Michael and L. Simon, Sobolev and mean-value inequalities on generalized submanifolds of $\mathbb{R}^{n}$, Comm. Pure Appl. Math. 26 (1973), 361-379.

[S-Y] R. Schoen and S.-T. Yau, Harmonic maps and the topology of stable hypersurfaces and manifolds with non-negative Ricci curvature., Comment. Math. Helv. 51 (1976), no. 3, 333-341.

[V] N. Varopoulos, Potential theory and diffusion on Riemannian manifolds, Conference on harmonic analysis in honor of Antoni Zygmund, Vol. I, II (Chicago, Ill., 1981), 821-837, Wadsworth Math. Ser., Wadsworth, Belmont, CA, 1983.

Department of Mathematics, University of California, Irvine, CA 92697-3875, U.S.A.

E-mail address: pli@math.uci.edu

School of Mathematics, University of Minnesota, Minneapolis, MN 55455, U.S.A. E-mail address: jiaping@math.umn.edu 